\title{
La valoración que los graduados tienen sobre su formación universitaria desde su situación laboral actual ${ }^{1}$
}

\section{Graduates' assessment of their university education. An approach from their current employment status}

\author{
Albert SANCHEZ-GELABERT y José NAVARRO-CENDEJAS \\ Universitat Autònoma de Barcelona y \\ Centro de Investigación y Docencia Económica - CONACYT (México)
}

Recibido: Octubre 2014

Evaluado: Marzo2015

Aceptado: Marzo 2015

\begin{abstract}
Resumen
En este artículo analizamos la valoración que hacen los titulados universitarios sobre la formación recibida, así como la utilidad de ésta en su ocupación actual. El objetivo es analizar si existen diferencias en la valoración a partir del nivel formativo familiar y el sexo de los estudiantes. Además, examinamos si las condiciones laborales que tienen los titulados intervienen en la valoración de los estudios. Los resultados muestran la existencia de diferencias significativas entre los jóvenes según su origen social y sexo, siendo las mujeres y los jóvenes de clase trabajadora los que valoran mejor su formación recibida. Finalmente, con la introducción de la situación laboral como variable de control constatamos que, en algunos casos, estas diferencias son debidas al contexto laboral actual de los graduados más que a diferencias sociodemográficas.
\end{abstract}

Palabras clave: satisfacción con los estudios, educación superior, utilidad de la formación, situación laboral y educación.

\begin{abstract}
The present article analyzes graduates' evaluation of their training its usefulness in their current occupation. The aim is to analyze if there are differences in the evaluation depending on students' gender and family educational background. In turn, the study addresses whether graduates' working conditions affect these evaluations. Results show the existence of significant differences between the young depending on their social origin and gender. Women and working class graduates are more likely to make a better assessment of their university
\end{abstract}

\footnotetext{
${ }^{1}$ Esta investigación forma parte del "Plan Nacional de investigación científica, desarrollo e investigación tecnológica" financiado por el Ministerio Español de Ciencia e Innovación, con el título "Itinerarios Universitarios, equidad y movilidad ocupacional" (CSO2010-19271).
} 
training. Finally, holding graduates' employment situation as a control variable, we verify that in some cases these differences are due to the current working environment of the graduates rather than to demographic differences.

Keywords: student satisfaction, higher education, usefulness of training, employment situation and education.

En el contexto de crisis económica actual, la adquisición de una titulación universitaria adquiere un significado importante para hacer frente a la vida laboral y evitar situaciones de precariedad en el futuro. La importancia de adquirir un nivel de estudios superiores se configura como una condición necesaria, aunque no suficiente, para poder acceder a un trabajo cualificado y evitar situaciones de riesgo de exclusión sociolaboral a lo largo de toda la trayectoria laboral de los individuos.

En las últimas décadas, la Universidad Española ha experimentado una serie de cambios que han derivado en un nuevo modelo universitario, pasando de un modelo elitista a un sistema de educación superior masivo. El incremento en el número de estudiantes y la heterogeneidad de la población universitaria, conjuntamente con las reformas llevadas a cabo en años recientes, han contribuido a repensar el modelo universitario y el papel que juega la universidad en la sociedad actual.

El aumento de la participación de estudiantes en la universidad se ha triplicado en los últimos 50 años, lo que ha supuesto una diversificación del perfil de estudiante con respecto al origen social, al sexo, a las experiencias educativas anteriores, así como a las motivaciones previas y a las expectativas de futuro (Troiano, 2005). Esto se traduce consecuentemente en diferentes formas de entender la experiencia universitaria, así como en la aparición de demandas diferenciadas por parte de los estudiantes (Ariño y Llopis, 2011; Troiano y Elias, 2014; Elias y Sanchez-Gelabert, 2014).

A estos cambios en la configuración del alumnado, se le suman reformas profundas que ha experimentado la universidad para hacer frente a las exigencias del proceso de globalización económica, que ha abierto la puerta a lo que se ha denominado como "sociedad de la información". A partir de la consideración de una universidad que intenta responder a las demandas del mercado laboral y de la importancia creciente de la adquisición de un título, el carácter profesionalizador de la universidad se convierte en un factor clave que tiene una influencia importante en la construcción del rol y la identidad de los actuales estudiantes universitarios, pero al mismo tiempo puede convertirse en un elemento fundamental de acceso a la universidad para las generaciones que están por llegar. Además, con la incorporación al Espacio Europeo Educación Superior, las universidades españolas han implementado cambios tanto en la estructura y oferta de las titulaciones como en las metodologías docentes, todo ello con la finalidad de fomentar la movilidad y la empleabilidad de la población universitaria.

En este sentido, el Consejo Europeo recomienda para el horizonte 2020 un aumento de la cobertura en educación superior, como una medida fundamental para fomentar el crecimiento económico, el aumento de puestos de trabajo cualificados y la emergencia de modelos productivos más sostenibles, teniendo como elementos fundamentales la 
innovación y el conocimiento. Concretamente, propone una reducción de la tasa del abandono de estudios universitarios hasta situarla por debajo del $10 \%$ y, a su vez, propone una tasa de graduación en educación superior en torno al $40 \%$ para los jóvenes entre 30 y 34 años (Consejo Europeo, 2009).

En este contexto, algunos autores han señalado que la complejidad creciente, tanto de la sociedad en general como de la misma universidad, ha contribuido a generar un tipo de estudiante relativamente desmotivado o poco motivado por los estudios que o bien abandona relativamente pronto o bien disminuye su participación y asistencia a las actividades académicas (Masjuan, 2004). Frente a esta diversidad del alumnado universitario en su composición, así como en sus expectativas, motivaciones y demandas, la importancia de indagar sobre la valoración que los estudiantes hacen de la formación recibida se convierte en un elemento central de evaluación del sistema universitario y de su papel en la sociedad actual.

Tomando en cuenta estas consideraciones, este artículo pretende analizar las valoraciones de los graduados cuatro años después de terminar sus estudios universitarios, teniendo en cuenta tanto factores sociodemográficos (sexo y origen social) como la experiencia laboral de los universitarios a partir de su graduación. En primer lugar, se analiza si existen patrones explicativos sobre la relación entre los factores sociodemográficos y la valoración de la universidad y, en segundo lugar, se indaga si esta valoración puede estar influida por el contexto laboral actual, es decir, si el éxito o fracaso en el mercado de trabajo (como indicador objetivo) puede tener un efecto en la valoración retrospectiva de la experiencia universitaria.

\section{La satisfacción con los estudios universitarios: estudios previos}

En una primera aproximación a la literatura relacionada con la valoración de los alumnos sobre su experiencia universitaria, en otras palabras su satisfacción con los estudios, encontramos que normalmente las investigaciones se centran en la etapa durante la cual los individuos están todavía dentro del sistema universitario como alumnos (Martin et al., 2000). En este sentido, aunque la satisfacción de los estudiantes es generalmente aceptada como una actitud a corto plazo que resulta de la experiencia educativa de los estudiantes (Elliot y Healy, 2001), algunos autores consideran que la incorporación de instrumentos que permitan al graduado evaluar la formación recibida en función de la ocupación, la empleabilidad y las habilidades que desarrolla en dicha ocupación, así como la evaluación de las aspiraciones en comparación con el trabajo logrado, puede ser más efectivo como indicador más amplio y global de valoración y de satisfacción (Harvey y Knight, 1996; Lees, 2002; Delaney, 2001; Gil et al., 2010).

La recopilación de información proporcionada por los graduados universitarios sobre su experiencia educativa es fundamental para la mejora del servicio que ofrecen las universidades, así como un mecanismo de evaluación de las reformas introducidas recientemente. Así, el estudio de la satisfacción de los estudiantes puede considerarse por un lado como un indicador de la manera en que las instituciones responden a las necesidades de los alumnos y, por otro, como una medida de la efectividad, éxito y vitalidad institucional (Low, 2000; Upcraft y Schuh, 1996). 
Además, el análisis de dicha información puede aportar elementos interesantes para intentar comprender la diversidad que observamos en el mundo universitario actual (Carot et al., 2011), sobre todo si asumimos que una de las principales dificultades al hablar de valoración del sistema universitario y de la formación recibida es su carácter multidimensional, tal y como han destacado diferentes autores (Hartman y Schmidt, 1995; Machado et al., 2011; García-Aracil, 2009).

Con respecto a la manera de abordar el estudio de la satisfacción de los universitarios, Aldemir y Gülcan (2004) proponen analizar cuatro factores que tienen un efecto en la satisfacción de la universidad: factores institucionales, factores extracurriculares, expectativas de los estudiantes y características sociodemográficas. Siguiendo esta propuesta, el objetivo del presente artículo es analizar dos de estas dimensiones. Por un lado, aspectos relacionados con las expectativas de los estudiantes $^{2} \mathrm{y}$, por otro, factores sociodemográficos, el sexo y el nivel formativo de los padres.

Por otro lado, diversos estudios han analizado los factores que influyen en la valoración de los estudiantes o de los graduados universitarios con respecto a sus estudios y a su aplicación en la vida post universitaria. En este sentido, Belfield et al. (1999) distinguen entre circunstancias previas y actuales; las primeras relacionadas con factores individuales, tales como sexo, origen social o la carrera estudiada, mientras que las circunstancias actuales hacen referencia a la situación laboral, a la composición de sus familias, al sector laboral o a la ubicación geográfica. En este artículo retomamos esta sugerencia y analizamos algunos de estos factores, tanto los previos como los actuales, con la finalidad de encontrar la influencia de ambos, así como la relación que existen entre ellos.

Con respecto al sexo, en una investigación con titulados de diferentes países europeos, García-Aracil (2009) encuentra que las mujeres están menos satisfechas con sus estudios que los hombres. Estos hallazgos coinciden con otros autores que también encuentran mayor insatisfacción con la universidad por parte de las mujeres (Birenbaum y Feldman, Drew y Work, en García-Aracil, 2009; Pike, 1994). Dichos autores justifican estos resultados bajo el argumento de que las mujeres se sienten relativamente menos privilegiadas $\mathrm{y}$, consecuentemente, tienden a sentirse menos satisfechas que sus compañeros varones. En cambio, otros autores (Adelman, en Martin et al., 2000; Belfied, 1999) consideran que esta relación se invierte siendo los hombres los que presentan mayores índices de insatisfacción, es decir, que las mujeres se sienten más satisfechas con los estudios realizados.

Con respecto al origen social de los estudiantes, se identificaron menos evidencias que permitan concluir cuál es su influencia. En el mismo estudio de García-Aracil (2009) referido anteriormente, se encontró que los titulados que provenían de orígenes formativos familiares más altos presentaban mayores niveles de insatisfacción con sus estudios que el resto de sus compañeros.

\footnotetext{
${ }^{2}$ En nuestro caso particular, utilizamos como proxi de expectativas la inserción laboral de los graduados, entendiendo una inserción consolidada como indicador de expectativas cumplidas.
} 
El tercer componente considerado es el impacto de la situación laboral después de haber concluido los estudios. En las investigaciones consultadas los temas que se han indagado corresponden al impacto de encontrarse trabajando, de las condiciones laborales o de la satisfacción con el trabajo en la valoración retrospectiva de la formación universitaria. Así, en la investigación de Belfied et al. (1999), se concluyó que una variable importante en la valoración de la universidad era la situación en la que los graduados se encontraban con respecto al trabajo. Los resultados indican que aquellos que no se encontraban trabajando tenían una visión negativa sobre la contribución de la educación superior hacia el hecho de ser productivos con la sociedad, o bien, aquellos que trabajaban por cuenta propia tenían una impresión menos favorable acerca de la contribución de sus estudios con respecto a asegurarse un ingreso. Según estos autores, la valoración de los graduados sobre su formación, al ser una evaluación sobre el pasado, está influenciada por la situación actual. Sin embargo, la situación laboral -o en general vital- de los titulados es producto de muchas circunstancias, entre las cuales evidentemente tendrá algún peso el paso por la universidad, pero distinto para cada titulado en función de sus circunstancias particulares.

Por su parte, Pike (1994) encontró que en general aquellos graduados que estaban más satisfechos con sus trabajos, eran más propensos reportar satisfacción con las experiencias educativas universitarias, fenómeno que denomina "efecto halo". Volviendo al punto tocado anteriormente, sobre la diferenciación según sexo, es importante reconocer que la satisfacción con el trabajo de las mujeres suele diferenciarse de la de los hombres, principalmente respecto a las diferencias en ingresos. Así, controlando por la satisfacción laboral, Pike encontró que la insatisfacción con el ingreso tenía un efecto más significativo en la calificación de la experiencia para las mujeres que para los hombres.

Finalmente, García-Aracil (2009) muestra que quienes tienen puestos de trabajo no propios del nivel universitario, reportan peores índices de satisfacción. Además, aquellos titulados que ocupan lugares de trabajo relacionados con su especialidad están más satisfechos que los que trabajaban en ocupaciones no relacionadas con sus estudios.

\section{Modelo de análisis e hipótesis}

Se propone un modelo de análisis que tiene como objetivo analizar si existen diferencias entre la valoración de la formación recibida y su utilidad según el sexo y el origen formativo familiar de los estudiantes. A su vez, tal y como se plantea en la Figura 1, se pretende contrastar si la situación laboral de los graduados interviene en la valoración de la universidad teniendo en cuenta las variables sociodemográficas mencionadas. Así, nos interesa contrastar si las condiciones y características de la ocupación laboral de los estudiantes afectan diferencialmente a la valoración de la formación recibida y la utilidad de esta según el sexo y el nivel formativo familiar de los estudiantes. 


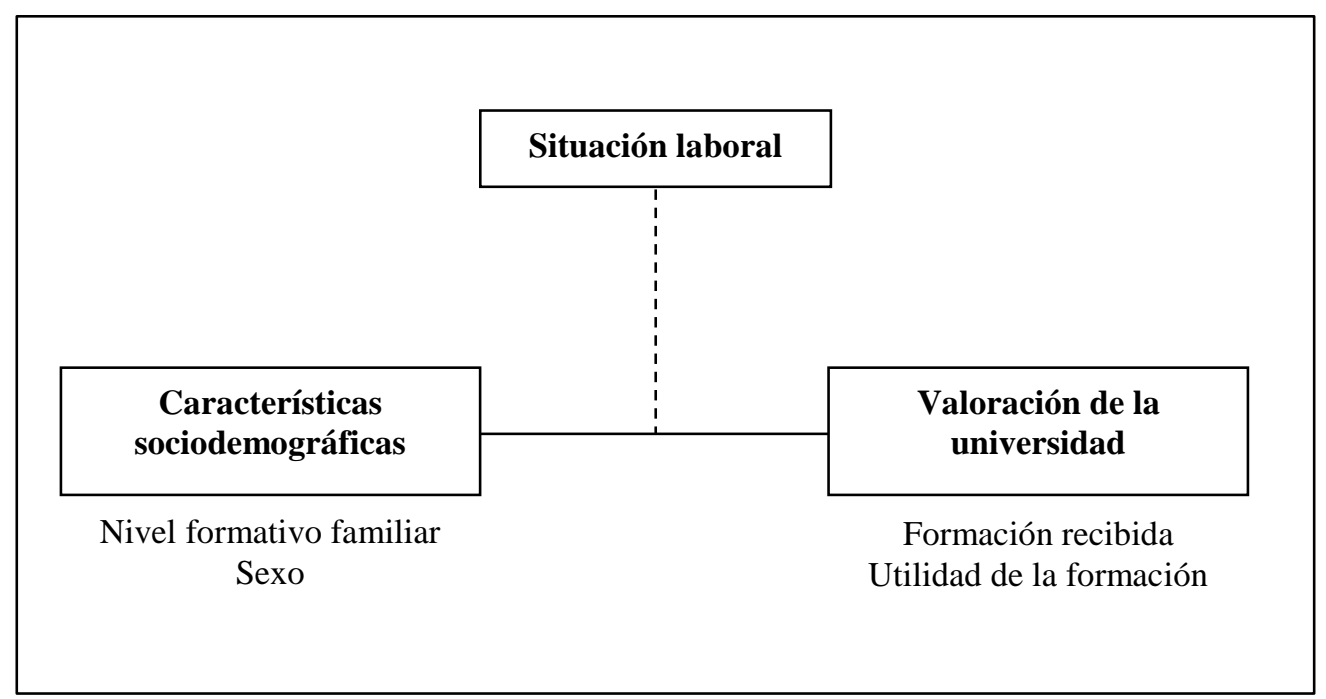

Figura 1.Modelo de análisis

\section{Hipótesis}

1. El nivel formativo familiar tendrá una influencia en la valoración de los titulados de la formación universitaria recibida así como en la valoración de su utilidad para el trabajo:

a. Los estudiantes de orígenes familiares inferiores tenderán a valorar mejor la formación recibida y su utilidad.

b. Los jóvenes que provienen de familiar con niveles formativos superiores valoraran peor su experiencia universitaria -en términos de formación y utilidad para el empleo.

2. El sexo de los estudiantes afectará la valoración que estos hacen de la formación y de la utilidad:

a. Las mujeres valorarán más positivamente la universidad.

3. Las hipótesis presentadas anteriormente $(\mathrm{H} 1$ y H2) se transformarán en función del tipo de inserción laboral de los estudiantes. Por tanto, el tipo de situación laboral tendrá un efecto en la valoración de la universidad y la utilidad de la formación para el trabajo:

a. Inserciones más precarias tendrán una valoración inferiores de la formación y su utilidad, independientemente del origen formativo familiar y del sexo de los estudiantes.

b. En el caso de inserciones laborales más exitosas los estudiantes valorarán más positivamente su formación universitaria y la utilidad, sin distinción de origen social o sexo. 


\section{Metodología y datos}

Los datos utilizados para realizar el análisis provienen de la Cuarta Encuesta de Inserción Laboral de los graduados de las universidades catalanas, elaborada por la Agencia para la Calidad del Sistema Universitario de Cataluña (AQU Catalunya, 2011). La encuesta fue llevada a cabo a inicios de 2011, con egresados de las universidades catalanas de la promoción 2006-2007. Se tomó la decisión de analizar estos datos ya que, de acuerdo con diferentes autores, este tipo de encuestas constituyen un elemento privilegiado para medir el grado de satisfacción con los estudios y a su vez se convierten en herramientas con mucho potencial en la gestión universitaria (Cabrera et al., 2003; Mora y Vidal, 2005; Delaney, 2008).

Para esta investigación se conformó una muestra que incluyera únicamente a los titulados menores de 36 años. Esta selección responde a la necesidad de centrar el análisis en la población más joven y evitar posibles sesgos relacionados con los colectivos de mayor edad que acceden a la universidad con experiencias laborales previas y cuya participación y características difieren significativamente de la mayoría de titulados.

Con el mismo objetivo, se tomó en cuenta únicamente a titulados de las universidades públicas catalanas. Aunque pueden existir diferencias según la titularidad de la universidad, la intención del presente artículo no es una comparación entre universidades sino que se pretende analizar la valoración retrospectiva de la formación y su utilidad de los titulados teniendo en cuenta su situación laboral tres años después de abandonar la universidad. En este sentido, nos hemos concentrado en los titulados de universidades públicas, que corresponden al $80 \%$ del total de encuestados por AQU Catalunya, para evitar sesgos en la valoración de la universidad en función del costo financiero que implica la asistencia a una universidad privada.

\section{Resultados}

\section{Valoración de la formación recibida y de su utilidad}

En este artículo medimos la valoración de los egresados de la universidad a partir de dos indicadores. El primero se refiere a la valoración de los titulados de la formación que recibieron en la universidad, mientras que el otro consiste en la evaluación de esa formación con respecto a su aplicabilidad en el mundo del trabajo. Para ello, se llevó a cabo un análisis factorial teniendo en cuenta 11 de los 14 ítems originales de la encuesta ${ }^{3}$, mediante el cual pudimos diferenciar cinco factores ${ }^{4} \mathrm{de}$ valoración tanto de la formación universitaria como de la utilidad: competencias

\footnotetext{
${ }^{3}$ El cuestionario se puede consultar en la página web de AQU Catalunya : www.aqu.cat/estudis

${ }^{4}$ Se realizó un análisis factorial de manera exploratoria para poder reducir y recodificar la información de los datos originales. A partir del análisis se pensó que era oportuno eliminar 3 ítems - pensamiento crítico, creatividad y habilidades de documentación- que reducían la significatividad y la fiabilidad de los factores y dificultaban la comprensión de algunas dimensiones.
} 
comunicativas (expresión oral y escrita), competencias transversales (trabajo en equipo, liderazgo, resolución de problemas, toma de decisiones, gestión), competencias instrumentales (idioma e informática), formación teórica y formación práctica.

Finalmente, la construcción de las variables valoración de la formación universitaria y valoración de la utilidad de la formación se hizo a partir de la mediana de las puntuaciones obtenidas en las respuestas del cuestionario y no de la escala original de valoración (que va del uno como la peor valoración al siete como la mejor).

\begin{tabular}{lll}
\hline & \multicolumn{2}{c}{ Valoración } \\
Ítems del cuestionario & $\alpha$ cronbach & Utilidad \\
\hline Expresión oral & & \\
Comunicación escrita & & \\
Trabajo en equipo & \\
Liderazgo & \\
Resolución de problemas & \\
Toma de decisiones & $\mathbf{0 , 8 5}$ & \\
Gestión & & \\
Informática & & \\
Idiomas & \\
Formación teórica & \\
Formación práctica &
\end{tabular}

Tabla 1. Análisis de fiabilidad de los ítems relacionados con la valoración universitaria

Analizando la valoración de la formación universitaria recibida, en la Tabla 2 observamos que existe una relación estadística significativa según el sexo de los estudiantes, siendo las mujeres las que hacen una valoración más alta de la formación recibida en la universidad que los hombres (54,6\% frente a 46,8\%). Algo similar sucede con la valoración de la utilidad de la formación recibida para el empleo (Tabla 3): las mujeres valoran más alto que los hombres la utilidad de la formación, con más de 6 puntos porcentuales de diferencia. De esta manera, podemos afirmar, como se planteaba en la hipótesis 2 , que las mujeres tienen una valoración más alta de la formación recibida y de la utilidad de esta para el trabajo.

\begin{tabular}{cccc}
\hline & Mujer & Hombre & Total \\
\hline Baja & $45,4 \%$ & $53,2 \%$ & $48,4 \%$ \\
Alta & $54,6 \%$ & $46,8 \%$ & $51,6 \%$ \\
\hline Total & $100 \%$ & $100 \%$ & $100 \%$ \\
\hline
\end{tabular}

Tabla 2. Valoración de la formación recibida por sexo de los graduados. Nota: $\mathrm{p} \leq 0,05$ para la prueba chi-cuadrado. 


\begin{tabular}{cccc}
\hline & Mujer & Hombre & Total \\
\hline Baja & $42,4 \%$ & $48,7 \%$ & $44,8 \%$ \\
Alta & $57,6 \%$ & $51,3 \%$ & $55,2 \%$ \\
\hline Total & $100 \%$ & $100 \%$ & $100 \%$ \\
\hline
\end{tabular}

Tabla 3. Valoración de la utilidad de la formación recibida por sexo de los graduados. Nota: $\mathrm{p} \leq$ 0,05 para la prueba chi-cuadrado.

Centrando el análisis en la relación entre nivel formativo familiar y valoración de la universidad, observamos que existe una relación significativa entre el origen de los estudiantes y la valoración de la formación recibida. Siendo los jóvenes que provienen de orígenes formativos más altos los que tienen una valoración inferior de la formación recibida (47\%) que sus compañeros de orígenes medios y bajos $(53,6 \%$ y 54,1\%, respectivamente).

\begin{tabular}{ccccc}
\hline & Bajo $^{\mathbf{5}}$ & Medio & Superior & Total \\
\hline Baja & $45,9 \%$ & $46,4 \%$ & $53,0 \%$ & $48,4 \%$ \\
Alta & $54,1 \%$ & $53,6 \%$ & $47,0 \%$ & $51,6 \%$ \\
\hline Total & $100 \%$ & $100 \%$ & $100 \%$ & $100 \%$
\end{tabular}

Tabla 4. Valoración de la formación recibida por nivel formativo familiar de los graduados.

Nota: $\mathrm{p} \leq 0,05$ para la prueba chi-cuadrado.

Es destacable, a su vez, evidenciar que referente a la utilidad de la formación no se han encontrado diferencias significativas entre el origen formativo de los estudiantes y la valoración de la utilidad del conocimiento recibido para el lugar de trabajo. Así, se puede afirmar que la hipótesis planteada con relación a la valoración y el nivel formativo familiar (H1) se cumple a medias. Es decir, se acepta para la valoración de la formación recibida pero no existe relación significativa entre el origen formativo familiar y la valoración de la utilidad de la formación en el ejercicio profesional.

\section{La situación laboral de los graduados catalanes}

En primer lugar presentamos algunos datos descriptivos sobre la situación laboral de los graduados universitarios de las universidades públicas catalanas (Tabla 5). Los resultados muestran un alto índice de ocupación, 87,5\%, frente a las situaciones de paro e inactividad, $8,8 \%$ y $3,8 \%$ respectivamente.

\footnotetext{
${ }^{5}$ Medido como el máximo nivel formativo alcanzado por cualquiera de los padres del titulado y clasificado como Bajo cuando el máximo nivel alcanzado por los padres es primario, Medio cuando al menos alguno de los dos llegó hasta estudios secundarios y Superior, cuando alguno de los padres obtuvo algún título de nivel universitario.
} 


\begin{tabular}{lcc}
\hline Situación Laboral & $\mathbf{n}$ & $\mathbf{\%}$ \\
\hline Ocupados & 8944 & 87,3 \\
Parados & 957 & 8,8 \\
Inactivos & 436 & 3,8 \\
\hline Total & $\mathbf{1 0 3 3 7}$ & $\mathbf{1 0 0}$ \\
\hline
\end{tabular}

Tabla 5. Situación laboral de los graduados universitarios

El desglose de la tabla anterior según sexo (Tabla 6), muestra que el porcentaje de mujeres es ligeramente superior en el caso de ocupados e inactivos. En contra, son los hombres los que presentan un porcentaje mayor en las situaciones de paro $(9,7 \%$ frente a $8,2 \%)$.

\begin{tabular}{lccc}
\cline { 2 - 3 } & \multicolumn{2}{c}{ Sexo } & \multirow{2}{*}{ Total } \\
\cline { 2 - 3 } & Mujer & Hombre & \\
\hline Ocupados & $87,8 \%$ & $86,6 \%$ & $87,3 \%$ \\
\hline Parados & $8,2 \%$ & $9,7 \%$ & $8,8 \%$ \\
\hline Inactivos & $4,0 \%$ & $3,7 \%$ & $3,8 \%$ \\
\hline Total & $100 \%$ & $100 \%$ & $100 \%$ \\
\hline
\end{tabular}

Tabla 6. Situación laboral de los graduados universitarios según sexo

Con respecto al nivel formativo familiar de los titulados (Tabla 7) se observa que los estudiantes que provienen de niveles inferiores (bajo y medio) están más presentes en situaciones de ocupación. Los estudiantes con un nivel formativo familiar superior presentan porcentajes más elevados en situaciones de paro e inactividad ${ }^{6}$.

\begin{tabular}{lcccc}
\cline { 2 - 4 } & \multicolumn{3}{c}{ Nivel formativo familiar } & \multirow{2}{*}{ Total } \\
\cline { 2 - 4 } & Bajo & Medio & Superior & \\
\hline Ocupados & $88,3 \%$ & $88,5 \%$ & $85,4 \%$ & $87,4 \%$ \\
\hline Parados & $8,4 \%$ & $7,8 \%$ & $10,1 \%$ & $8,8 \%$ \\
\hline Inactivos & $3,4 \%$ & $3,7 \%$ & $4,5 \%$ & $3,8 \%$ \\
\hline Total & $100 \%$ & $100 \%$ & $100 \%$ & $100 \%$ \\
\hline
\end{tabular}

Tabla 7. Situación laboral de los graduados universitarios por nivel formativo familiar

\footnotetext{
${ }^{6}$ Una de las explicaciones a esta diferencia viene dada por la continuación de otros estudios (mayoritariamente de tercer ciclo) de los estudiantes que provienen de familias con más formación.
} 


\section{Tipología de ocupaciones}

Uno de los objetivos de este artículo es analizar si la situación actual -referida a la ocupación- tiene un efecto en la valoración de la formación universitaria. Por lo tanto, a partir de este apartado analizamos únicamente a aquellos graduados que se encontraban ocupados en el momento de la encuesta. Para poder distinguir las diferentes situaciones laborales que muestran los titulados ocupados, procedimos a construir un análisis tipológico, a partir de la combinación de dos técnicas estadísticas multivariadas: Análisis de Correspondencias Múltiples (ACM) y Análisis de Clasificación (ACL) (Lebart et al., 2004).

De las variables que proporciona la Encuesta de Inserción Laboral de AQU Catalunya, se seleccionaron aquellas que permiten clasificar a los titulados de acuerdo a su ocupación, tomando en cuenta las dimensiones que proponen algunos autores para identificar situaciones de precariedad laboral juvenil (Rodgers 1989; Duell, 2004): dimensión temporal y organizacional, económica y social ${ }^{7}$. Complementariamente, se incorporó el contenido intelectual de la ocupación -solicitud o no del título universitario y realización de funciones de nivel profesional- como un indicador de la calidad en la inserción laboral de los jóvenes, tal como lo proponen otros autores (Santamaría, 2009). De esta manera, las variables elegidas para clasificar a los titulados de acuerdo a su ocupación, que para efectos del ACM se consideran variables activas, son las siguientes:

- Tipo de contrato: fijo (F), autónomo (A), temporal de larga duración -mayor de un año- (TLD, o temporal de corta duración -menor de un año-(TCD).

- Tipo de jornada laboral: completa (C) o parcial (P).

- Ingresos: de 9 mil o menos a 12 mil euros anuales brutos (9-12), de 12 a 24 mil (12-24) o de 24 a 40 mil o más (24-40).

- Duración del trabajo actual: hasta un año (1), de dos a cuatro años (2-4) o más de cinco años (5).

- Requisito para el trabajo: requiere título universitario o no ${ }^{8}$.

- Funciones realizadas en el trabajo: propias del nivel universitario o no.

Después de aplicar las técnicas estadísticas seleccionadas, se obtuvieron cuatro tipos, cuyas características se sintetizan en la Tabla 8, de acuerdo a las categorías que mejor los definen. A continuación mencionamos algunos rasgos que caracterizan a cada uno de los cuatro tipos.

\footnotetext{
${ }^{7}$ Las dimensiones organizacional y social, no han podido ser introducidas en el análisis tipológico ya que la base de datos no proporciona información relacionada con estos factores.

${ }^{8}$ Aunque la encuesta separa entre el requisito del título específico o cualquier título universitario, se han clasificado en una misma categoría, porque en este trabajo no nos interesa valorar la adecuación concreta entre un título y el puesto de trabajo, sino la diferenciación entre un trabajo que requiere estudios universitarios del resto de trabajos con menor exigencia intelectual.
} 


\begin{tabular}{|l|l|l|l|l|l|l|l|l|l|l|l|l|l|l|l|l|l|}
\hline \multirow{2}{*}{$\begin{array}{c}\text { Tipos de } \\
\text { inserción }\end{array}$} & \multicolumn{3}{|c|}{ Tipo de contrato } & $\begin{array}{c}\text { Jornada } \\
\text { laboral }\end{array}$ & \multicolumn{3}{|c|}{ Ingresos } & \multicolumn{2}{c|}{$\begin{array}{c}\text { Duración } \\
\text { trabajo }\end{array}$} & \multicolumn{2}{c|}{$\begin{array}{c}\text { Requisito } \\
\text { de título }\end{array}$} & $\begin{array}{c}\text { Funciones } \\
\text { propias }\end{array}$ \\
\hline & F & A & TLD & TCD & C & P & $9-12$ & $12-24$ & 2440 & 1 & 24 & 5 & Sí & No & Sí & No \\
\hline Consolidación & & & & & & & & & & & & & & & \\
\hline $\begin{array}{l}\text { Aproximación } \\
\text { sucesiva }\end{array}$ & & & & & & & & & & & & & & & & \\
\hline $\begin{array}{l}\text { Tray. larga } \\
\text { duración }\end{array}$ & & & & & & & & & & & & & & & & \\
\hline Precariedad & & & & & & & & & & & & & & & \\
\hline
\end{tabular}

Tabla 8. Características principales de los tipos de inserción laboral.

Tipo 1: Consolidación. Se trata de titulados que han tenido un cierto nivel de éxito en su inserción laboral después de la graduación. Es decir, a los pocos años de haber egresado de la Universidad ya se encuentran en posiciones que se podrían considerar estables desde un punto de vista laboral, tomando en cuenta los indicadores seleccionados y la comparación con el resto de titulados.

Tipo 2: Aproximación sucesiva. Hemos nombrado aproximación sucesiva a este grupo de acuerdo a Casal et al. (2006), quienes proponen una tipología para clasificar a los jóvenes, de acuerdo a sus trayectorias vitales. Dentro de esta categoría los autores incluyen a aquellos jóvenes que apuntan hacia trayectorias de éxito, pero que han tenido que pasar por diferentes estadios entre los estudios y el trabajo antes de lograr cierta estabilidad. Esta clasificación toma en cuenta a la totalidad de jóvenes, mientras que en nuestro estudio solo abordamos a los titulados universitarios, quienes de alguna manera ya forman parte de una élite que ha conseguido llegar hasta el final de la educación formal. Sin embargo, tomamos el símil de la clasificación porque en nuestro grupo de aproximación sucesiva estamos incluyendo a titulados que, aunque ya tienen rasgos de cierto éxito ocupacional, todavía no logran consolidarse: tienen poco tiempo en el trabajo actual, con contratos temporales y no reportan los ingresos más altos comparativamente a otros titulados.

Tipo 3: Trayectoria de larga duración. Este grupo conjunta a los titulados que tuvieron una inserción laboral previa a la titulación, tomando en cuenta que tenían más de cinco años en el trabajo y la mayoría no tuvo que presentar un título universitario para conseguir su trabajo actual. Lo interesante aquí es que buena parte de estos titulados continúan realizando funciones que no son propias del nivel universitario.

Grupo 4: Precariedad. Se trata de los titulados que, en comparación con el resto, presentan las peores condiciones de trabajo. Es decir, al poseer un título universitario no se podrían comparar con los jóvenes en situación de precariedad de los que hablan Casal et al. (2006), más bien hablamos de una precariedad relativa al resto de titulados.

En la Tabla 9 se puede observar que casi la mitad de los graduados se clasifican dentro del grupo de consolidados $(47,1 \%)$, es decir, se trata de jóvenes que presentan una cierta estabilidad laboral. El tipo de aproximación sucesiva, comprende un 23,2\% 
de los graduados. Este grupo de jóvenes tienen situaciones laborales con menor estabilidad que los anteriores, ya que han durado poco tiempo en el trabajo y tienen contratos mayoritariamente temporales, aunque poseen trabajos que podrían ser calificados como propios del nivel universitario. Finalmente, cerca de un tercio de los graduados $(29,7 \%)$ fueron clasificados en tipos de inserciones de larga duración y de precariedad. Si vemos a los tipos desde una perspectiva jerárquica de calidad de inserción laboral, este tercio correspondería a los graduados con peores niveles de inserción, aunque con diferencias importantes entre ambos grupos. Por un lado, los de larga duración se caracterizan por haberse mantenido por más de cinco años en el puesto de trabajo, con contratos fijos, pero con un nivel de ingresos inferior al del grupo de consolidados, probablemente debido a que las funciones que realizan no son propias del nivel universitario; mientras que por su parte, los que tienen inserción precaria trabajan a jornada parcial, no realizan funciones propias del nivel universitario, tienen contratos temporales y en consecuencia a lo anterior, su nivel de retribución es inferior al de cualquiera de los otros tres tipos.

\begin{tabular}{lcc}
\hline Tipo de Inserción laboral & $\mathbf{n}$ & $\mathbf{\%}$ \\
\hline Consolidados & 4212 & 47,1 \\
Aproximación sucesiva & 2076 & 23,2 \\
Inserción larga duración & 1512 & 16,9 \\
Precariedad & 1144 & 12,8 \\
\hline Total & 8944 & 100 \\
\hline
\end{tabular}

Tabla 9. Tipología de titulados ocupados

\section{El efecto de la situación ocupacional en la valoración de los estudios}

De acuerdo al modelo de análisis propuesto, una de las hipótesis de esta investigación era analizar cómo la situación ocupacional tiene un efecto en la valoración que los graduados hacen de su formación recibida y de la utilidad de ésta. Siguiendo esta línea argumental, se incorporó la tipología de situaciones ocupacionales previamente construida para ver la relación entre las variables, realizando un análisis de contingencia trivariado.

En primer lugar observamos que los titulados con situaciones laborales consolidadas o en fase de consolidación -aproximación sucesiva-, valoran más positivamente la formación recibida que en el caso de precariedad laboral o de inserción de larga duración (53,1\% y $54,2 \%$ frente a $47,8 \%$ y $48,3 \%$ respectivamente). Esta constatación, muestra cómo la situación laboral afecta a la valoración de la formación recibida de forma que en situaciones de mayor estabilidad ocupacional se valora mejor la formación recibida.

Las diferencias valorativas según el sexo de los titulados se mantienen en los tipos consolidados e inserción de larga duración, existiendo una valoración superior de la formación recibida $(53,1 \%$ y 54,2\%). En cambio, para los tipos de situación laboral de 
aproximación sucesiva y precariedad, a pesar de conservar el patrón mujer-mayor valoración, no existen diferencias significativas entre la valoración y el sexo. Así, vemos cómo la situación laboral influye en las relaciones sexo-valoración de la formación, de manera que en ciertas situaciones laborales (precariedad laboral y aproximación sucesiva) las diferencias por sexo se ven compensadas.

\begin{tabular}{lccc}
\hline \multirow{2}{*}{ Tipología Laboral } & \multicolumn{2}{c}{ Valoración alta según sexo } & \multirow{2}{*}{ Total } \\
& Mujer & Hombre & \\
\hline Consolidados* & $57,0 \%$ & $48,1 \%$ & $53,1 \%$ \\
\hline Aproximación sucesiva ** & $55,7 \%$ & $51,1 \%$ & $54,2 \%$ \\
\hline Inserción larga duración* & $52,3 \%$ & $42,3 \%$ & $47,8 \%$ \\
\hline Precariedad ** & $49,9 \%$ & $43,8 \%$ & $48,3 \%$ \\
\hline
\end{tabular}

Tabla 10. Valoración alta de la formación recibida por tipología laboral y sexo de los graduados. Nota: * $\mathrm{p} \leq 0,05$ para la prueba khi-cuadrado; ** No significativo.

Al analizar la valoración de la utilidad de la formación, las diferencias entre categorías laborales se ahondan más profundamente. Mientras los titulados con inserciones consolidadas o en proceso de consolidación valoran mejor la utilidad de la formación, aquellos en situaciones de precariedad o que se conservan el trabajo en el que se insertaron antes de finalizar la titulación (larga duración), muestran que la formación recibida no ha sido útil para las ocupaciones que realizan. Estas diferencias indican que cuando la formación no resulta útil para poder consolidar su situación laboral o acceder a lugares de trabajo relacionados con la formación recibida tienen una baja percepción de la utilidad de la formación.

\begin{tabular}{|c|c|c|c|}
\hline \multirow{2}{*}{ Tipología laboral } & \multicolumn{2}{|c|}{ Valoración Alta según sexo } & \multirow{2}{*}{ Total } \\
\hline & Mujer & Hombre & \\
\hline Consolidados* & $64,0 \%$ & $59,2 \%$ & $61,9 \%$ \\
\hline Aproximación sucesiva* & $64,2 \%$ & $55,4 \%$ & $61,4 \%$ \\
\hline Inserción larga duración* & $39,9 \%$ & $31,2 \%$ & $36,0 \%$ \\
\hline Precariedad $* *$ & $45,5 \%$ & $40,1 \%$ & $44,0 \%$ \\
\hline
\end{tabular}

Tabla 11. Valoración alta de la utilidad de la formación recibida por tipología laboral y sexo de los graduados. Nota: $* \mathrm{p} \leq 0,05$ para la prueba khi-cuadrado; ** No significativo

Lo que resulta más destacable es contrastar que las diferencias por sexo en la valoración de la utilidad desaparecen en ciertas situaciones laborales. Así, mientras se conserva el binomio mujeres-mayor valoración de la utilidad en 3 de las categorías de 
tipología laboral, esta no muestra diferencias significativas para los jóvenes con inserciones de carácter más precario.

De esta manera, se aceptan parcialmente las hipótesis planteadas. Por un lado, se confirma que en las peores situaciones laborales los titulados muestran peor valoración con relación a la formación recibida y su utilidad sin que existan diferencias por sexo (H3a). En cambio, en las inserciones laborales más exitosas la valoración en los dos aspectos analizados sí muestra diferencias significativas según la tipología laboral y el sexo de los graduados (H3a), siendo las mujeres las que refuerzan la valoración positiva.

Con relación al nivel formativo de origen y la valoración de los titulados, las relaciones bivariadas mostraban una asociación entre orígenes formativos más altos y una valoración inferior de la formación recibida. Al analizar de qué manera la tipología laboral actúa sobre esta asociación se constata un patrón similar a los antes mencionados con relación al sexo. En las categorías más exitosas de tipología laboral, es decir, los estudiantes consolidados o en vías de consolidación (aproximación sucesiva) se conserva la relación significativa mencionada; siendo los titulaciones de orígenes más bajos (bajo y medio) los que mejor valoran la formación recibida. En cambio, en las inserciones laborales de más precariedad o de larga duración, no existen diferencias significativas entre el origen formativo y la valoración de la formación recibida.

\begin{tabular}{lcccc}
\hline \multirow{2}{*}{ Tipología Laboral } & \multicolumn{4}{c}{ Valoración alta según nivel formativo familiar } \\
& Bajo & Medio & Superior & Total \\
\hline Consolidados* & $56,5 \%$ & $54,1 \%$ & $48,4 \%$ & $53,1 \%$ \\
\hline Aproximación sucesiva* & $58,4 \%$ & $55,6 \%$ & $48,9 \%$ & $54,2 \%$ \\
\hline Inserción larga duración ** & $48,5 \%$ & $50,8 \%$ & $43,0 \%$ & $47,8 \%$ \\
\hline Precariedad ** & $50,7 \%$ & $50,3 \%$ & $44,7 \%$ & $48,2 \%$ \\
\hline
\end{tabular}

Tabla 12. Valoración alta de la formación recibida por tipología laboral y nivel formativo familiar de los graduados. Nota: $* \mathrm{p} \leq 0,05$ para la prueba khi-cuadrado; ** No significativo

Finalmente, con respecto a la utilidad de la formación, la incorporación de la tipología laboral nos permite analizar cambios en las relaciones con el nivel formativo familiar. Para los titulados que tienen situaciones laborales de aproximación sucesiva, se mantiene la relación entre mayor formación familiar y menor valoración. En cambio, en todos los demás tipos de situaciones laborales, se diluyen las diferencias valorativas por origen formativo familiar, mostrando diferencias estadísticas no significativas. 


\begin{tabular}{lcccc}
\hline \multirow{2}{*}{ Tipología Laboral } & \multicolumn{4}{c}{ Valoración alta por nivel formativo familiar } \\
& Bajo & Medio & Superior & Total \\
\hline Consolidados ** & $61,3 \%$ & $62,7 \%$ & $61,5 \%$ & $61,8 \%$ \\
\hline Aproximación sucesiva* & $64,4 \%$ & $62,1 \%$ & $57,7 \%$ & $61,3 \%$ \\
\hline Inserción larga duración ** & $36,1 \%$ & $36,6 \%$ & $34,8 \%$ & $35,9 \%$ \\
\hline Precariedad ** & $44,7 \%$ & $45,4 \%$ & $42,2 \%$ & $43,9 \%$ \\
\hline
\end{tabular}

Tabla 13. Valoración alta de la utilidad de la formación recibida por tipología laboral y nivel formativo familiar de los graduados. Nota: $* \mathrm{p} \leq 0,05$ para la prueba khi-cuadrado; $* *$ No significativo

A la luz de estos resultados, podemos contrastar las hipótesis planteadas con relación al efecto de la situación laboral de los estudiantes (H3), analizando la valoración de la formación y su utilidad y las variables sociodemográficas elegidas. Así pues, se acepta la primera hipótesis (H3a) donde se planteaba que las inserciones más precarias tendrían una valoración inferior de la formación y su utilidad sea cual sea el origen formativo familiar y el sexo de los estudiantes. En contra, sólo se puede aceptar parcialmente en el caso de inserciones laborales más exitosas (H3b). En el caso de los titulados consolidados, se diluyen las diferencias por origen formativo referidas a la valoración de la utilidad de la formación. Contrariamente a lo planteado, sí que se encuentran diferencias significativas según el origen formativo familiar y cómo valoran los jóvenes la formación recibida siendo los que tienen orígenes formativos familiares más altos los que la valoran peor.

\section{Conclusiones e implicaciones}

El planteamiento inicial que se proponía en este artículo era analizar la valoración de los titulados sobre la formación recibida y sus utilidad para el trabajo y, a su vez, si el tipo de ocupación mostraba diferencias según el sexo y el nivel formativo familiar de origen. A la luz de los resultados, podemos afirmar que existe una relación entre el sexo y el nivel formativo de los titulados y su valoración de la universidad.

Como se ha visto en nuestros resultados, la valoración de los estudios universitarios y de su aplicación en el mundo laboral tiene diferencias de acuerdo a las características sociodemográficas analizadas. Una de las posibles explicaciones a los resultados encontrados en este artículo puede estar relacionada con las desigualdades referentes al acceso a la universidad (en términos de clase social) y a las situaciones de desigualdad de condiciones laborales (particularmente con relación al sexo). Según este argumento, los jóvenes en peores condiciones tenderán a priori a valorar mejor la formación universitaria, ya que funciona como un mecanismo de minimización de las posibles dificultades y desigualdades que pueden encontrar en el mercado laboral.

Con respecto a la situación laboral de los titulados, los resultados muestran que cuando la formación no resulta útil para poder consolidar su situación laboral o acceder a lugares de trabajo con correspondan al nivel de formación recibida, los titulados 
tienden a tener una baja percepción de la utilidad de ésta. Así, consideramos que en el contexto de crisis económica actual, las dificultades de inserción y la precarización de las condiciones laborales pueden generar un sentimiento de frustración y de mala valoración o insatisfacción entre los titulados con la formación recibida y su utilidad al ver que no se alcanzan las expectativas profesionalizadoras y laborales que tenían al acceder a la universidad.

Por otro lado, algunos autores ya apuntaban que los titulados satisfechos con la universidad tenderán a promover en su entorno más cercano las ventajas de estudiar una carrera y además en una determinada institución y especialidad (Martin et al., 2000). Este mismo "efecto halo" (Pike, 1994) podría darse en sentido negativo si la frustración e insatisfacción de los titulados desencadena en un sentimiento generalizado de inutilidad de la formación universitaria que no es capaz de responder a las demandas del mercado de trabajo.

En este sentido, aunque es importante considerar que la universidad es una institución que no explica por sí sola los resultados que sus titulados obtienen en el mercado de trabajo, si éste no responde a las expectativas de los graduados se pueden generar dinámicas de desprestigio de los estudios universitarios, con consecuencias que pueden impactar a futuras generaciones en el proceso de elección de acceder o no a la educación superior. Esto es particularmente importante en épocas de crisis como la actual, a la cual hay que añadir el componente de recortes en el gasto público en educación.

Quedan por explorar en futuras investigaciones otras dimensiones relacionadas con la satisfacción de los titulados con sus estudios. Por ejemplo, el efecto diferencial que existe entre titulaciones, que es una de las variables que mejor explica las diferencias en los resultados que obtienen los universitarios en el mercado de trabajo. Otra dimensión consiste en la comparación entre las valoraciones que tienen los universitarios durante la etapa de formación frente a la que muestran una vez que terminaron los estudios y se enfrentan al mercado de trabajo. Este tipo de estudios longitudinales requiere de una fuerte inversión en tiempo y recursos, pero los resultados pueden generar procesos que mejoren la formación al interior de las universidades. Asimismo, en futuras investigaciones será importante comparar los resultados encontrados aquí con el colectivos de titulados que provienen de universidades privadas, cuyas motivaciones y valoraciones podrían diferir en función del costo financiero que implica acceder a ese tipo de instituciones.

\section{Referencias bibliográficas}

ALDEMIR, C. y GULCAN, Y. (2004). Student satisfaction in higher education. Higher Educational Management and Policy, 16, 109-122.

AQU CATALUNYA (2011). Universitat i treball a Catalunya 2011. Barcelona: Agència per a la Qualitat del Sistema Universitari de Catalunya. 
ARIÑO, A. y LLOPIS, R. (2011). ¿Universidad sin clases? Condiciones de vida de los estudiantes universitarios en España (Eurostudent IV). Madrid: Ministerio de Educación, Cultura y Deporte.

BELFIELD, C.R., BULLOCK, A. D., y FIELDING, A. (1999). Graduates' views on the contribution of their Higher Education to their general development: A Retrospective Evaluation for the United Kingdom. Research in Higher Education, 40(4), 409-438.

CABRERA, A.F., WEERTS. D.J., y ZULICK, B.J. (2003). Encuestas a egresados: tres fundamentos conceptuales en el seguimiento de egresados universitarios, en VIDAL, J. (coord.) Métodos de análisis de la inserción laboral de los universitarios. Leon: Consejo de Coordinación Universitaria y Universidad de León.

CAROT, J., CONCHADO, A., MORA, J. y VILA, L. (2011). La opinión de los graduados europeos sobre la universidad cinco años después de haber finalizado sus estudios. Papers, 96 (4), 1269-1285.

CONSEJO EUROPEO (2009). Council conclusions of 12 May 2009 on a strategic framework for European cooperation in education and training ('ET 2020'). JOC, $119(2)$.

DELANEY, A. (2001). Assessing Undergraduate Education from Graduating Seniors' Perspective: Peer Institutions Provide the Context. Tertiary Education and Management, 7(3), 255-276.

DELANEY, A. (2008). Typical institutional research studies on students: Perspective and examples. New Directions for Higher Education, 141, 57-67.

DUELL, N. (2004). Defining and assessing precarious employment in Europe: a review of main studies and surveys. Recuperado de: http://www.economix.org, $10 / 12 / 2013$.

ELIAS, M. y SANCHEZ-GELABERT, A. (2014). Relación entre actitudes y acciones de aprendizaje de los estudiantes universitarios. Revista de Estudios E Investigación En Psicología Y Educación, 1(1), 3-14.

ELLIOT, K. M., y HEALY, M. A. (2001). Key factors influencing student satisfaction related to recruitment and retention. Journal of Marketing for Higher Education, 10 (4), 1-11.

GARCÍA-ARACIL, A. (2009). European graduates' level of satisfaction with higher education. Higher Education, 57, 1-21.

GIL, J., GARCÍA, E., y SANTOS, C. (2010). Miradas retrospectivas de los egresados sobre la educación superior. Revista de Investigación Educativa, 27(2), 371-393.

HARTMAN, D. E., y SCHMIDT, S. L. (1995). Understanding student/alumni satisfaction from a consumer's perspective: The effects of institutional performance and program outcomes. Research in Higher Education, 36(2), 197-217. 
HARVEY, L. y KNIGHT, P. (1996) Transforming Higher Education. Buckingham: Open University Press and Society for Research into Higher Education.

LEES, D. (2002). Graduate Employability - Literature Review. Recuperado de: http://www.qualityresearchinternational.com/esecttools/esectpubs/leeslitreview.pdf, 26/03/2013.

LEBART, L., MORINEAU, A., y PIRON, M. (2004). Statistique exploratoire multidimensionnelle. Paris: Dunod.

LOW, L. (2000). Are college students satisfied? A national analysis of changing expectations. Indianapolis: USA Group Foundation.

MACHADO, M., BRITES, R., MAGALHAES, A. y SÁ, M. (2011). Satisfaction with Higher Education: critical data for student development. European Journal of Education, 46(3), 415-432.

MARTIN, A., MILNE-HOME, J., BARRET, J., SPALDING, E. y JONES, G. (2000). Graduate satisfaction with university and perceived employment preparation. Journal of education and work, 13(2), 199-213.

MASJUAN, J.M. (2004). Convergencia europea, reformas universitarias, actitudes y prácticas de los estudiantes. Educar, 33, 59-76.

MORA, J.-G. y VIDAL, J. (2005), The emerging uses of alumni research in Spain. New Directions for Institutional Research, 2005(126) 73-82.

PIKE, G. R. (1994). The Relationship Between Alumni Satisfaction and Work Experiences. Research in Higher Education, 35(1), 105-123.

RODGERS, G. (1989): 'Precarious work in Western Europe: The state of the debate", en Rodgers and Rodgers, comp. Precarious Jobs in Labour Market Regulation: The Growth of Atypical Employment in Western Europe. Bruselas: International Institute for Labour Studies and Free University of Brussels.

SANTAMARÍA, E. (2009). Precariedad laboral: apuntes para una aproximación sociológica a sus formas contemporáneas. Papeles del CEIC. Recuperado de: http://redalyc.uaemex.mx/src/inicio/ArtPdfRed.jsp?iCve=76512777007, 26/03/2012.

TROIANO, H. (2005). Consistencia y orientación del perfil profesional, percepción del mercado laboral y reacciones previstas ante posibles dificultades de inserción. Papers, 76, 167-197.

TROIANO, H., y ELIAS, M. (2014). University access and after: explaining the social composition of degree programmes and the contrasting expectations of students. Higher Education, 67(5), 637-654.

UPCRAFT, M. L., y SCHUH, J. H. (1996). Assessment in student affairs: A guide for practitioners. San Francisco: Jossey-Bass. 


\title{
Correspondencia con los autores
}

\author{
Albert SANCHEZ-GELABERT \\ Departamento de Sociología \\ Facultad de Ciencias Políticas y Sociología. \\ Universitat Autònoma de Barcelona \\ 08193 - Bellaterra \\ e-mail: albert.sanchez@uab.cat \\ José NAVARRO-CENDEJAS \\ Centro de Investigación y Docencia Económicas \\ Edificio de Investigación Nivel -1 \\ Carretera México-Toluca 3655 \\ Colonia Lomas de Santa Fe, Delegación Álvaro Obregón \\ 01210, México D.F, México \\ e-mail: jose.navarro@cide.edu
}

